\title{
Assessing Error in Human Dental Measurements: A Comparison of Resin Casts, Plaster Casts, and Dental Enamel
}

\author{
Amelia R. Hubbard ${ }^{1,2 *}$, Natasha Wilson ${ }^{2}$, Giuseppe Vercellotti ${ }^{3}$ \\ ${ }^{1}$ Anthrotech, Inc.; Yellow Springs, Ohio \\ ${ }^{2}$ Department of Sociology and Anthropology, Wright State University \\ ${ }^{3}$ Department of Anthropology, The Ohio State University
}

Keywords: methods, dental anthropology, dental casts, measurement error

\begin{abstract}
Technical advances in 3D morphometrics and other forms of digital analysis allow for detailed measurements of dental metrics yet, consistently, dental anthropologists show a publishing preference for measurements using dental calipers. For many researchers, dental casts are often measured when field seasons or collections-based trips do not allow ample time to measure the original teeth. As such, this study aimed to assess differences among measurements of plaster casts, resin casts, and dental enamel to determine if variables such as material softness or shrinkage could lead to measurement error. Results of a paired t-test demonstrate no statistically significant difference in buccolingual crown measurements from 23 commingled canines and first molars. Likewise, while plaster casts exhibited overall smaller mean (and individual) measurements than enamel and resin, the differences (around $0.04 \mathrm{~mm}$ on average) are negligible. We, therefore, conclude that casts can be used in place of original teeth, where needed, and which material type is "best" can be determined by the researcher's preferred medium.
\end{abstract}

Often circumstances do not allow for the long-term use of skeletal material for research purposes (e.g., repatriation and reburial, length of research visit, etc.). In such cases, ongoing availability of materials, through the production of dental impressions (negative replica of the teeth) and dental casts (lifelike reproductions from impressions) is more practical. However, in order to collect accurate measurement data, differences in error rates between casted dentition and the original teeth must be statistically insignificant so that the results of the study are not impacted. As such it is imperative that a researcher knows whether dental replicas consistently over or underestimate dental dimensions compared to dental enamel, and whether there are significant differences in measurements between casting mediums. Similarly, it is crucial to know whether the tools used to measure replicas impact precision.

The dimensional stability of the materials used to make dental impressions and dental casts has been widely studied. Dental alginates (irreversible hydrocolloid compounds) have long been favored as an impression material in clinical settings for their low cost. This material is not often used by dental anthropologists because it does not have long-term dimensional stability requiring casts to be made within a few hours or a single day (e.g., Sedda, Casarotto, Raustia, and Borracchini, 2008). In skeletally based dentitions there is also a higher chance that teeth will be pulled from the jaw given the density of the material and need for a dental tray to hold the alginate. Within dental anthropology, the use of polyvinyl siloxane replica materials (PVS) has become more common because it is gentler on skeletal dentitions and multiple casts can be made from the same impression as needed, due to long-term dimensional stability (e.g., Chee and

${ }^{*}$ Correspondence to:

Amelia Hubbard

Anthrotech, Inc.

E-mail: amy@anthrotech.net 
Donovan, 1992) as long as temperatures do not fluctuate wildly (Kelso, Hulsey, and Driscoll, 2020). Casting material such as gypsum plaster (plaster) are popular when dental morphometrics are the focus, though epoxy resins (resin) have gained popularity among dental anthropologists due to the higher resolution of surface features such as linear enamel hypoplasia and macrowear/ microwear features. A recent study of commonly used resin and plaster materials found that there was no difference in measured size between epoxy resins and dental stone, though the study measured differences using a Scanning Electron Microscope (Junior, Kreve, and Carvalho, 2018).

While dental anthropologists can measure casts using a variety of tools ranging from calipers to $3 \mathrm{D}$ scans (e.g., Al-Mulla and Murad, 2010; Bowes, Dear, Close, and Freer, 2017; Keating, Knox, Bibb, and Zhurov, 2008; Kumar, Phillip, Kumar, Rawar, Priya, Kumaran, 2015; Reuschl, Wieland, Stiesch, Wenzel, and Dittmer, 2016), calipers are still widely used across different field and lab settings. In measurements using calipers material surface hardness is a primary concern as the metal tips must be tightly fit to the surface of the replica and have the potential to leave impressions or scratches on soft surfaces. The most commonly used caliper for measuring dental dimensions is the HillsonFitzgerald caliper (Hillson, FitzGerald, and Finn, 2005). These calipers are made of a durable metal with thin needle point tips for better fit in interproximal spaces of in-situ dentitions (i.e., teeth implanted in the jaw). Of the three dental surfaces, enamel is the hardest and should be unaffected by the adjustment of the metal calipers. Resin is also a durable material that is unlikely to yield surface stability, but still softer than dental enamel. In contrast, plaster is soft and can be more easily depressed if the calipers are fit too tightly, though visible inspection of a plaster cast can clarify if the surface is damaged (i.e., a small depression would be readily visible).

When previous studies are analyzed to ascertain answers to the questions posed in our study, differences in approach, temporal changes in the quality of impression and casting products, and emergent technologies for measuring dentitions make comparisons problematic. For example, early studies such as Hunter and Priest (1960) measured dental stone casts taken from alginate impressions and enamel (intra-orally in living patients) using a Helios-style caliper (i.e., no needle tips). Later studies such as Pant, Juszczyk, Clark, and Radford (2008) use PVS impressions materials to make plas- ter casts to compare with both $3 \mathrm{D}$ printed resins (measured by a scanning electron microscope) and digital scans (measured using computer software). Further, the conditions in which impressions are taken can have quite an impact. For example, Hollinger, Lorton, Krantz, and Connelly (1984) found "material distortion and shrinkage is unpredictable" (308) in impressions of edentulous individuals and highly impacted by the preparation of the dental tray while Pant et al. (2008) found that temperature differences impacted the "architectural stability" of PVS. Studies such as Kelso et al. (2020) demonstrate additional concerns about the impacts of temperatures on long-term storage of materials.

This study examines the two most common casting materials (epoxy resin and dental stone) measured using the most common field and lab instrument (Hillson-Fitzgerald calipers) to assess differences in buccolingual dimensions. Assuming careful measurements of each replica's surface (e.g., checking that the calipers did not leave a visible surface indentation) and demonstrated low surface shrinkage rates of the materials measured, we predicted that buccolingual dimensions of both the plaster and resin casts would not be significantly different than the original dental enamel. However, given the softness of plaster casts we predicted that measurements would consistently register as smaller than either resin or plaster.

\section{Materials and Methods}

The specimens used in this study were collected from an ossuary located in Chiavari, Italy in the region of Liguria. The ossuary contains the remains of several hundred individuals who were removed from mausoleums and placed in a secondary burial pit as sanctioned by Italian law (DPR n.285/1990). With the exception of a limited number of individuals with identifying materials, the remains deposited in the ossuary are commingled and no contextual information is readily available. Based on cemetery records, the majority of the remains belong to individuals who lived in Chiavari and its hinterland between the late nineteenth and early twentieth century.

Twenty-three canine and first molar crowns were measured following Hillson et al. (2005). Dental impressions were made using a highresolution polyvinyl siloxane compound (Affinis), preferred by dental anthropologists because of its dimensional stability. These dentition were selected based on larger samples of preserved teeth and, thus, represent a convenience sample. Casts were made of gypsum plaster (Dentstone) and an epoxy 


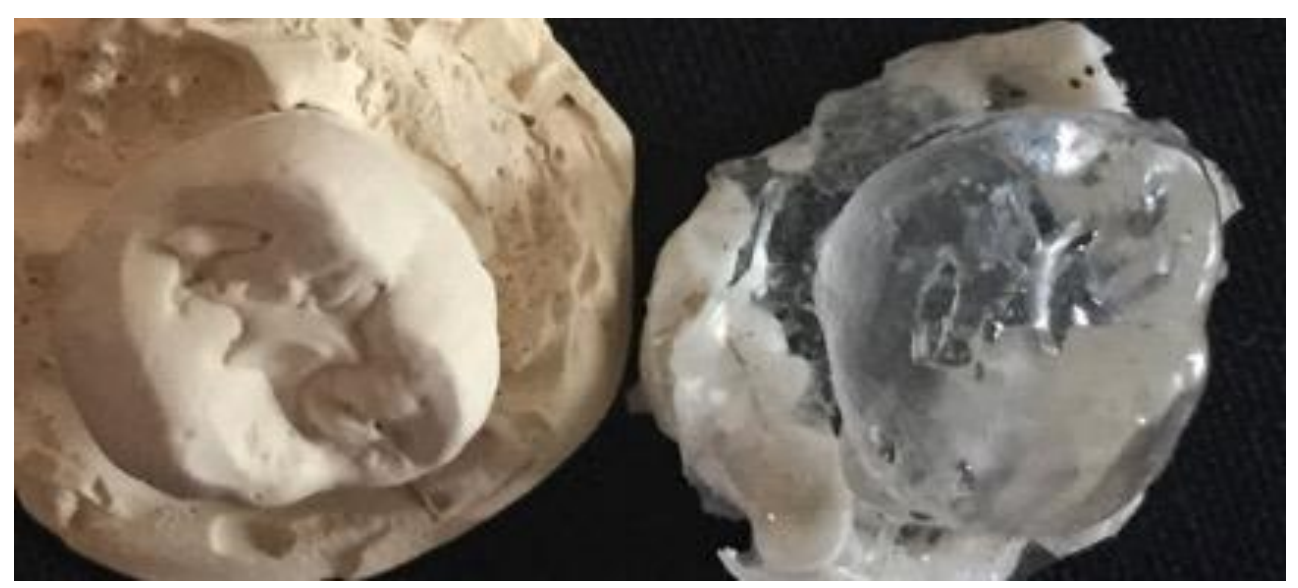

Figure 1: Plaster (left) and resin (right) casts of a first molar.

resin (Epofix) (Figure 1). The company Modern Materials produces Dentstone and reports a 0.11 percent "Expansion" rate of materials. The company Struers produces Epofix and claims a 0.3 percent shrinkage rate. Dental dimensions were recorded by a single researcher to eliminate interobserver error using Paleo-Tech's digital HillsonFitzergerald calipers (Hillson et al., 2005), which automatically import measurements into a designated Excel spreadsheet when a button is depressed on the caliper's surface. To reduce intraobserver error, dimensions of each tooth were recorded five times consecutively and reported as an average.

Only buccolingual crown dimensions were measured in this study because this dental measurement is unaffected by mixed samples of isolated teeth (i.e., not in situ) and those still imbedded in the jaw with neighboring teeth (i.e., in situ). Conversely, mesiodistal measurements are more challenging to measure because of tight interproximal spacing between teeth in-situ. Therefore, when trying to measure between interproximal spaces, there is a higher likelihood that resin or epoxy would be impressed by the tips of the calipers (and affect measurement). As the sample comprised both loose teeth and those imbedded in the jaw, this posed an increased chance that significant differences in measurements would reflect differences in applied pressure to the cast surface. Therefore, because this study used a mixed sample, measurements of mesiodistal differences on the tooth surfaces would not have been comparable. Further while comparative analyses between mesiodistal measurements for isolated teeth and in-situ dentitions was possible, overall small samples sizes available in the study did not allow for such a fine scale analysis of the cause of potential measure- ment variation.

To test the first proposal, that buccolingual crown dimensions would not vary significantly between enamel, resin, and plaster, a paired t-test was used (following Kieser, 1990). A paired t-test examines mean differences between groups of data and is reported as a t-value and associated significance or p-value (in this case with a $95 \%$ confidence interval). To test the second proposal, that buccolingual measurements of plaster would be smaller, simple mean differences were compared as well as individual measurements to determine the proportion of cases in which plaster measured as smallest. Though small sample sizes were used in the present study, statistical power would not be improved by a larger sample because the variables are not dependent.

\section{Results and Discussion}

Table 1 presents the results of the paired $t$-test comparing buccolingual measurements on tooth enamel to plaster and resin casts. None of the pairings demonstrated a statistically significant difference in measurement at the 0.05 (95\%) confidence interval. These results support our predictions that both types of casts can be used in lieu of the original teeth when conducting odontometric analyses, without significant error in measurement.

Table 2 reports the mean measurements for each material type. Measurements of the enamel were slightly larger on average than either plaster or resin, though plaster casts (as predicted) had the smallest mean of the three. Still, the differences between the overall means were minimal. Enamel measured, on average, $0.035 \mathrm{~mm}$ larger than resin and $0.043 \mathrm{~mm}$ larger than plaster. In the raw data, there were only three cases $(13 \%)$ in which one or more of the replicas (plaster or resin) and/or origi- 
Table 1. Paired t-test results comparing tooth enamel, plaster casts, and resin casts (* indicates statistical significance).

\begin{tabular}{lccl}
\hline Material type & $\mathrm{N}$ & $\mathrm{t}$ & $\mathrm{p}$-value \\
\hline Enamel-Resin & 23 & 0.8985 & 0.3787 \\
Enamel-Plaster & 23 & 1.0464 & 0.3068 \\
Plaster-Resin & 23 & 0.9751 & 0.3401 \\
\hline
\end{tabular}

nal tooth (enamel) measured exactly the same. Interestingly, individal measurements of plaster casts were lowest in more than half the cases (52\%), followed by enamel $(22 \%)$, and resin $(13 \%)$. Therefore, while plaster replicas produced some of the smallest measurements they were not exclusively the smallest measurements. Overall, despite measurable differences between individual teeth and in the combined sample, for all intents and purposes these measurements are identical.

The primary limitations of this study are sample size and composition. While the overall collection was estimated at 600 individuals, only a small portion of the collection resides in the U.S. Additionally, differential preservation, significant tooth wear, and other limiting variables in the roughly 60 individuals meant only 23 teeth were able to be measured. Additionally, this sample was one of convenience because it was available and nearby to the university where the study took place; therefore, these teeth may not reflect dental crown variability that could impact measurement. Similarly, as a modern human sample we are unable to conclude that such measurements are similarly accurate for other primate dentition, where there may be higher morphological variability. Finally, as noted in the materials and methods section, only buccolingual crown dimensions were gathered. While it was easy to measure isolated teeth in this setting, we were unable to replicate in situ dentition (i.e., teeth seated in the maxilla and/or mandible). Such measurements require a higher level of dexterity to measure because of potentially tight interproximal spacing. As such, there is always the possibility that heavier compression of the calipers on softer cast materials could have resulted in significant differences between measurements.

Given the minor differences between measurements, material choice can be determined by the researcher's preference because both casting materials provide accurate measurements. The researchers found that plaster casts were generally easier to measure than either enamel or resin casts because resin is translucent and has a smooth surface texture (like dental enamel) while plaster is opaque
Table 2. Mean measurements and proportions $(n=23)$.

\begin{tabular}{lcc}
\hline Material type & $\begin{array}{c}\text { Mean } \\
\text { (in mm) }\end{array}$ & $\begin{array}{c}\text { Proportion } \\
\text { (in \%) }\end{array}$ \\
\hline Enamel & 10.242 & 22 \\
Plaster & 10.199 & 52 \\
Resin & 10.207 & 13 \\
\hline
\end{tabular}

and slightly textured. Specifically, the texture of plaster makes it less likely that caliper tips slip during measurements. If cost is an issue, resins also tend to be more expensive than dental plasters.

\section{Conclusions}

The results of this study provide solid support for the continued use of either plaster or resin dental casts as proxies for original teeth, when needed. Future research might examine different tooth types, different primate and ancestral hominin species, and/or include mesiodistal measurements. The present study excluded incisors and premolars, therefore, additional research on measurement error rates for incisors or premolars could be examined to determine if the results were specific to canines and molars. Additionally, wide variation in tooth morphology and size across primate and ancestral hominin species could produce issues with measurement that should be considered.

\section{REFERENCES}

Al-Mulla, A.A., \& Murad, S.M. (2010). Accuracy and measurements made on digital and study models (a comparative study). Mustansiria Dental Journal, 7(1), 71-82.

Bowes, M., Dear, W., Close, E., \& Freer, T.J. (2017). Tooth width measurement using the Lythos digital scanner. Australasian Orthodontic Journal, 33(1), 73-81.

Chee, W.W.L., \& Donovan, T.E. (1992). Polyvinyl siloxane impression materials: a review of properties and techniques. Journal of Prosthetic Dentistry, 68, 728-32.

Hillson, S., FitzGerald, C., \& Flinn, H. (2005). Alternative dental Measurements: Proposals and relationships with other measurements. American Journal of Physical Anthropology, 126, 413426.

Hollinger, J.O., Lorton, L., Krantz, W.A., \& Connelly, M. (1984). A clinical and laboratory comparison of irreversible hydrocoloid impression techniques. Journal of Prosthetic Dentistry, 51, 304-309.

Hunter, W.S., \& Priest, W.R. (1960). Errors and discrepancies in measurement of tooth size. Jour- 
nal of Dental Research, 39, 405-408.

Junior, E.V.D.S., Kreve, S., \& Carvalho, G.A.P.D.

(2018). Analysis of linear dimensional change of different materials used for casting dental models: plaster type 4 , nanocomposites carbon nanostructures, polyurethane resin and epoxy resin. Journal of Dental Health, Oral Disorders $\mathcal{E}$ Therapy, 9(2), 200-205.

Keating, A.P., Knox, J., Bibb, R., \& Zhurov, A.I. (2008). A comparison of plaster, digital and reconstructed study model accuracy. Journal of Orthodontics, 35(3), 191-201.

Kelso, R.S., Hulsey, B.I., \& Driscoll, K.R.D. (2020). Dental molding compounds and casts: use in non-laboratory environments. Dental Anthropology, 33(1), 17-22.

Kieser, J.A. (1990). Human adult odontometrics: the study of variation in adult tooth size. New York, NY: Cambridge University Press.

Kumar, A.A., Phillip, A., Kumar, S., Rawat, A., Priya, S., \& Kumaran, V. (2015). Digital model as an alternative to plaster model in assessment of space analysis. Journal of Pharmacy and Bioallied Science, 7(S2), S465-469.

Pant, R., Juszczyk, A.S., Clark, R.K.F., \& Radford, D.R. (2008). Long-term dimensional stability and reproduction of surface detail of four polyvinyl siloxane duplicating materials. Journal of Dentistry, 36(6), 456-461.

Reuschl, R.P., Wieland, H., Stiesch, M., Wenzel, D., \& Dittmer, M.P. (2016). Reliability and validity of measurements on digital study models and plaster models. European Journal of Orthodontics, 38(1), 22-26.

Sedda, M., Casarotto, A., Raustia, A., \& Borracchini, A. (2008). Effect of storage time on the accuracy of cast made from different irreversible hydrocolloids. Journal of Contemporary Dental Practice, 9(4), 59-66. 